Nuclear Physics B95 (1975) 135-147

(c) North-Holland Publishing Company

\title{
NEUTRAL CURRENTS AND THE HIGGS MECHANISM
}

\author{
D,A. ROSS and M. VELTMAN \\ Institute for Theoretical Physics, University of Utrecht *
}

Received 11 April 1975

The consequences of assuming (i) weak and e.m. forces constitute a gauge field theory, and (ii) there are no heavy leptons, are investigated. Relative to the Weinberg model, introduction of a general spontaneous symmetry breaking system leads to a theory with one extra free parameter, namely the neutral vector boson mass. Experimental consequences are indicated. A particular Higgs system containing two multiplets is studied in detail. It is noted that parameters may be chosen such that the cosmological constant is zero before as well as after spontaneous symmetry breakdown.

\section{l. Introduction}

The idea that weak and e.m. interactions constitute a gauge theory has become quite popular with the discovery of neutral currents in neutrino reactions. To what extent the observed reaction rates coincide with the predictions of the Weinberg model is not yet clear, and as a matter of fact the situation is not entirely comfortable in this respect. At this point it seems very natural to ask what the consequences would be if deviations of the Weinberg model are observed. It is essentially this question that is considered in this paper.

Thus, suppose that the observed experimental reaction rates differ from the Weinberg model predictions. We then have the following options:

(i) Gauge theories do not apply;

(ii) There exist heavy leptons;

(iii) The spontaneous symmetry breaking mechanism is different from that of the Weinberg model.

In this paper we wish to consider possibility (iii). With respect to the experimental situation the conclusion is then that in general the mass of the neutral vector boson must be taken as a free parameter. This is discussed explicitly in sect. 5 of this paper.

Let us now describe things in somewhat more detail. In the model proposed by Glashow in 1961 [1] weak vector bosons and the photon are unified in an $\mathrm{SU}(2) \times \mathrm{U}(1)$ scheme, including a mixing angle $\theta_{\mathrm{W}}$ also used later by Weinberg.

* Postal address: Sorbonnelaan 4, Utrecht, The Netherlands. 
In the Glashow model the vector boson masses are put in by hand, and there is no relation between these vector boson masses and the mixing angle $\theta_{\mathrm{w}}$. But the algebra of the currents, i.e. the V-A structure of the neutral currents, is that of a gauge theory without heavy leptons. Weinberg, in his well-known paper [2], used the same structure for leptons and vector bosons, but introduced the spontaneous symmetry breaking of Higgs et al. [3] to generate the vector boson masses. In particular, only one Higgs multiplet of the lowest non-trivial representation was assumed, and this restriction leads to a relation between the vector boson masses involving the mixing angle $\theta_{\mathrm{w}}$. As observed by Weinberg *, the chief difference between his theory and that of Glashow is that the latter has less definite predictions. In fact, for experimental purposes the difference is that in the Weinberg model the mass of the neutral vector boson is fixed relative to the charged masses if the mixing angle is known.

However, there is really no rational reason why only the lowest Higgs representation would be present. First of all, from an experimental point of view the Higgs particles are essentially non-existent, and no limit as to the verification of their existence has been offered or is even suggested by the theory. Moreover, one may have ones doubts with respect to the Higgs mechanism, and in fact, a clear contradiction with experiment arises if one takes serious the cosmological constant arising from the Higgs scheme as applied in the Weinberg model [4]. For this and other reasons many theorists believe that the Higgs formulation is really something like an effective description of a much more complicated situation. Seen in this way there is really very little motivation for limiting oneself a priori to only the particular case as used in the Weinberg model, i.e. the introduction of only $I=\frac{1}{2}$ Higgs particles.

The logical thing to do seems therefore to return to Glashow's SU(2) $\times U(1)$ model, and then to supplement it with a spontaneous symmetry-breaking mechanism without any a priori limitation concerning the representation of the Higgs particles. It turns out that then the masses become essentially free parameters again, so that the predictive power of the model becomes equal to that of the Glashow model, at least if no direct experimental observation of the Higgs particles is involved.

It is natural to ask now whether the introduction of more Higgs multiplets relative to the case of the Weinberg model also helps with respect to the question of the cosmological constant. We have also investigated this, and the answer is affirmative.

In sect. 2 we will consider the special case of an admixture of $I=\frac{1}{2}$ and $I=1$ Higgs particles. With a given value for the mixing angle $\theta_{\mathrm{w}}$ the neutral vector boson mass may be varied between certain limits. In sect. 3 we examine the Higgs system in detail, and show that in this case no cosmological constant needs to arise from the spontaneous symmetry breakdown. That case seems to us a reasonable candidate for an effective description of a more involved situation as argued above. We

* Footnote 1 of ref. [2]. 
must however emphasize that the argument on the cosmological constant is not a hard argument and the difficulty can be cured by more conventional means. The case of a general mixture of Higgs scalars is considered in sect. 4, and the associated range of possible vector boson masses is indicated. Finally, the experimental consequences are discussed in sect. 5 .

\section{Admixtures of $I=\frac{1}{2}$ and $I=1$ Higgs particles}

In this article we will consequently use the tensorial notation for higher $I$-spin fields. The $I=1$ field is represented by a complex symmetrical tensor of rank two $\chi_{i j}$ or a traceless tensor $\chi_{i}^{j}$. Depending on the behaviour under the $\mathrm{U}(1)$ transformations there are two ways of assigning charges, namely one may have a self conjugate field with charges,- 0 and + , or a complex field with charges $0,-$ and - (and of course the conjugate charges $0,+$ and ++ ). In the first case we will use the traceless tensor while in the last case the symmetrical form will be used. In this way the behaviour with respect to the $U(1)$ part of the symmetry is most easily recognized.

Let us first consider the case where the $I$-spin 1 field is invariant under $U(1)$. Denoting the $I$-spin $\frac{1}{2}$ field by $\phi_{i}$ the part of the Lagrangian referring to the Higgs particles is:

$$
\begin{aligned}
& \mathcal{L}_{\mathrm{H}}=-\left(D_{\mu} \phi\right)^{+} D_{\mu} \phi-\mu \phi^{i} \phi_{i}-\frac{1}{2} \lambda\left(\phi^{i} \dot{\phi}_{i}\right)^{2} \\
& \quad-\left(D_{\mu} \chi\right)^{+} D_{\mu} \chi-\mu^{\prime} \chi_{j}^{i} \chi_{i}^{j}-\frac{1}{2} \lambda^{\prime}\left(\chi_{j}^{j} \chi_{i}^{j}\right)^{2} \\
& \quad+\frac{1}{2} a\left(\phi^{i} \phi_{i}\right)\left(\chi_{k}^{l} \chi_{l}^{k}\right)+\frac{1}{2} a^{\prime} \phi^{l} \phi_{i} \chi_{k}^{i} \chi_{l}^{k}+\frac{1}{2} b\left(\phi^{i} \phi_{j}-\frac{1}{2} \phi^{m} \phi_{m} \delta_{j}^{i}\right) \chi_{i}^{j}, \\
& \left(\phi_{i}\right)^{+}=\phi^{i}, \quad\left(\chi_{i}^{j}\right)^{+}=\chi_{j}^{i}, \\
& \left(D_{\mu} \phi\right)=\left\{\delta_{i}^{j} \partial_{\mu}+g W_{\mu}^{a}\left(-\frac{1}{2} i \tau^{a}\right)_{i}^{j}+g^{\prime} B_{\mu}\left(-\frac{1}{2} i \tau^{0}\right)_{i}^{j}\right\} \phi_{j}, \\
& \left(D_{\mu} \chi\right)_{i}^{j}=\left\{\delta_{k}^{j} \delta_{i}^{l} \partial_{\mu}+g W_{\mu}^{a}\left(\frac{1}{2} i \tau^{a}\right)_{k}^{j} \delta_{i}^{l}+g W_{\mu}^{a} \delta_{k}^{j}\left(-\frac{1}{2} i \tau^{a}\right)_{i}^{l}\right\} \chi_{l}^{k} .
\end{aligned}
$$

We note that we must have $\left(a+a^{\prime}\right)^{2}<4 \lambda \lambda^{\prime}$ so that the potential is always positive for large values of the fields.

In here, $\tau^{0}$ is the identity, and $g$ is related to $g$ through the Weinberg angle:

$$
g^{\prime}=-g s / c, \quad c=\cos \theta_{\mathrm{W}}, \quad s=\sin \theta_{\mathrm{W}}=e / g .
$$

$e$ and $g$ are the electric charge and the weak interaction coupling constant respectively. Numerically they are fixed by $e^{2} / 4 \pi=\frac{1}{137}$, and $g^{2} / 8 M^{2}=\sqrt{\frac{1}{2}} G$ where $M=$ charged vector boson mass, and $G=$ fermi constant $=1.02 \times 10^{-5} m_{\mathrm{p}}^{-2}$, with $m_{\mathrm{p}}=$ proton mass.

It is essential that in constructing this Lagrangian the anti-symmetrical tensors $e^{i j}$ are not to be used, because they are not invariant under the U(1) symmetry. 
The coupling of the Higgs particles to the physical neutral vector boson $Z_{\mu}$ and the e.m. field $A_{\mu}$ are obtained by substituting

$$
W_{\mu}^{3}=c Z_{\mu}-s A_{\mu}, \quad B_{\mu}=-s Z_{\mu}-c A_{\mu} .
$$

It is easily seen from eq. (3) that $\chi_{1}^{1}$ (and $\chi_{2}^{2}$ ) decouples not only from the e.m. field $A_{\mu}$ but also from the neutral meson field $Z_{\mu}$. Consequently, if $\chi_{1}^{1}$ (and $\chi_{2}^{2}$ ) develops a vacuum expectation value then only a contribution to the charged vector meson mass will result.

Of the three neutral fields (namely $\chi^{0}=\sqrt{\frac{1}{2}} \chi_{1}^{1}=-\sqrt{\frac{1}{2}} \chi_{2}^{2}$ and the real and imaginary parts of $\phi_{1}$ ) one becomes an unphysical Goldstone particle, while $\chi^{0}$ and the real part of $\phi_{1}$ remain physical, with arbitrary masses (with in general some mixing between them).

In view of the present experimental situation it is more interesting to consider the case of an $I$-spin 1 particle $\chi_{i j}$ that is not invariant under $\mathrm{U}(1)$.The Higgs Lagrangian becomes

$$
\begin{aligned}
& \mathcal{L}_{\mathrm{H}}=-\left(D_{\mu} \phi\right)^{+} D_{\mu} \phi-\mu \phi^{i} \phi_{i}-\frac{1}{2} \lambda\left(\phi^{i} \phi_{i}\right)^{2} \\
& -\left(D_{\mu} \chi\right)^{+} D_{\mu} \chi-\mu^{\prime} \chi^{i j} \chi_{i j}-\frac{1}{2} \lambda^{\prime}\left(\chi^{i j} \chi_{i j}\right)^{2} \\
& +\frac{1}{2} a\left(\phi^{k} \phi_{k}\right)\left(\chi^{i j} \chi_{i j}\right)+\frac{1}{2} a^{\prime} \phi^{i} \phi_{j} \chi^{j k} \chi_{i k} \\
& +\frac{1}{2} b \chi^{i j} \phi_{i} \phi_{j}+\frac{1}{2} b^{*} \chi_{i j} \phi^{i} \phi^{j} .
\end{aligned}
$$

The constant $b$ will be taken to be real. F urther

$$
\begin{aligned}
& \left(\chi_{i j}\right)^{+}=\chi^{i j}, \\
& \left(D_{\mu} \chi\right)_{i j}=\left\{\delta_{i}^{k} \delta_{j}^{l} \partial_{\mu}+g W_{\mu}^{a}\left(-\frac{1}{2} i \tau^{a}\right)_{i}^{k} \delta_{j}^{l}+g W_{\mu}^{a} \delta_{i}^{k}\left(-\frac{1}{2} i \tau^{a}\right)_{j}^{l}\right. \\
& \left.\quad+g^{\prime} B_{\mu}\left(-\frac{1}{2} i \tau^{0}\right)_{i}^{k} \delta_{j}^{l}+g^{\prime} B_{\mu} \delta_{i}^{k}\left(-\frac{1}{2} i \tau^{0}\right)_{j}^{l}\right\} \chi_{k l} .
\end{aligned}
$$

Now $\chi_{11}$ decouples from the e.m. field. Suppose $\chi_{11}$ develops a vacuum expectation value $H$. To make the equations transparent we introduce the matrix $w_{\mu}$ :

$$
w_{\mu}=-\frac{1}{2} i g W_{\mu}^{a} \tau^{a}-\frac{1}{2} i g^{\prime} B_{\mu} \tau^{0} .
$$

Substituting eq. (5) we find:

$$
w_{\mu}=-\frac{1}{2} i g\left(\begin{array}{cc}
(1 / c) Z_{\mu} & W_{\mu}^{1}-i W_{\mu}^{2} \\
W_{\mu}^{1}+i W_{\mu}^{2} & \left(\left(s^{2}-c^{2}\right) / c\right) Z_{\mu}+2 s A_{\mu}
\end{array}\right) .
$$

We have:

$$
\left(D_{\mu} \chi\right)_{i j}=\partial_{\mu} \chi_{i j}+w_{\mu i}^{k} \chi_{k j}+w_{\mu j}^{l} \chi_{i l} .
$$


If now

$$
\begin{array}{ll}
\chi_{i j}=H, & \text { if } i=j=1, \\
\chi_{i j}=0, & \text { otherwise }
\end{array}
$$

we find:

$$
\left(D_{\mu} \chi\right)_{i j}=H\left(w_{\mu i}{ }^{1} \delta_{j}{ }^{1}+w_{\mu j}{ }^{1} \delta_{i}{ }^{1}\right)
$$

or

$$
\begin{aligned}
& \left(D_{\mu} \chi\right)_{11}=-(i / c) g H Z_{\mu}, \\
& \left(D_{\mu} \chi\right)_{12}=\left(D_{\mu} \chi\right)_{21}=-\frac{1}{2} i g H\left(W_{1}+i W_{2}\right)_{\mu}, \\
& \left(D_{\mu} \chi\right)_{22}=0 .
\end{aligned}
$$

Thus

$$
\left|D_{\mu} \chi\right|^{2}=H^{2}\left(\left(g^{2} / c^{2}\right) Z^{2}+\frac{1}{2} g^{2} W_{1}^{2}+\frac{1}{2} g^{2} W_{2}^{2}\right) .
$$

The charged W mass gets a contribution $\mathrm{H}^{2} \mathrm{~g}^{2}$, and the neutral $\mathrm{Z}$ mass a contribution $2 H^{2} g^{2} / c^{2}$. For the $I$-spin $\frac{1}{2}$ field $\phi$ one finds similarly, using $\phi_{1}=F, \phi_{2}=0$ :

$$
\left(D_{\mu} \phi\right)_{1}=-(i / 2 c) g F Z_{\mu}, \quad\left(D_{\mu} \phi\right)_{2}=-\frac{1}{2} i g F\left(W_{1}+i W_{2}\right)_{\mu},
$$

and

$$
\left|D_{\mu} \phi\right|^{2}=\frac{1}{4} F^{2}\left(\left(g^{2} / c^{2}\right) Z^{2}+g^{2} W_{1}^{2}+g^{2} W_{2}^{2}\right) .
$$

Combined we find:

$$
M_{0}^{2}=\frac{2 H^{2} g^{2}}{c^{2}}+\frac{F^{2} g^{2}}{2 c^{2}}, \quad M_{ \pm}^{2}=H^{2} g^{2}+\frac{F^{2} g^{2}}{2} .
$$

In the case of the Weinberg model we have $H=0$. We then have:

$$
M_{0}^{2}=\left(1 / c^{2}\right) M_{ \pm}^{2} \text {. }
$$

In the case at hand with non-zero $H$ we get a larger $M_{0}^{2}$;for the extreme case that $F \simeq 0$

$$
M_{0}^{2}=\left(2 / c^{2}\right) M_{ \pm}^{2} .
$$

The above argument shows how with the addition of an $I$-spin 1 Higgs particle the mass of the neutral vector meson may be varied between 0 and $\sqrt{ } 2$ times the value given by the Weinberg model.

Returning now to the Lagrangian (6) we observe that it is possible to introduce $T$ violation by taking $b$ complex. If $b$ is real than one could in principle expect the possibility of a spontaneous breakdown of $T$ invariance. This and similar situations have been analyzed by Lee [5]; in this particular case it turns out that if $b$ is taken real no spontaneous breakdown of $T$ invariance can occur. 
Finally, it is worthwhile to note that if the coefficient $b$ is zero then the Lagrangian (6) has two independent global U(1) symmetries, namely with respect to $\phi$ and $x$ separately. After spontaneous breakdown two zero-mass neutral particles appear, of which one is an unphysical and the other a physical Goldstone boson. Furthermore this symmetry is not affected by the interaction of the Higgs particles with the vector bosons so that this physical Goldstone boson would remain massless to all orders [6]. A Higgs particle of such small mass that the associated force has a macroscopic range is experimentally excluded [4], and we conclude that the coefficient $b$ must be non-zero. Or, stated differentially, one must be careful not to have accidental symmetries in the Lagrangian that are subsequently spontaneously broken as the mechanism for obtaining vector-meson masses is put in action.

\section{The structure of the Higgs system}

The Higgs system is quite complicated, with a structure that deviates in several respects from the usually advocated simple systems. This difference is largely due to the occurrence of third-order terms in the interaction.

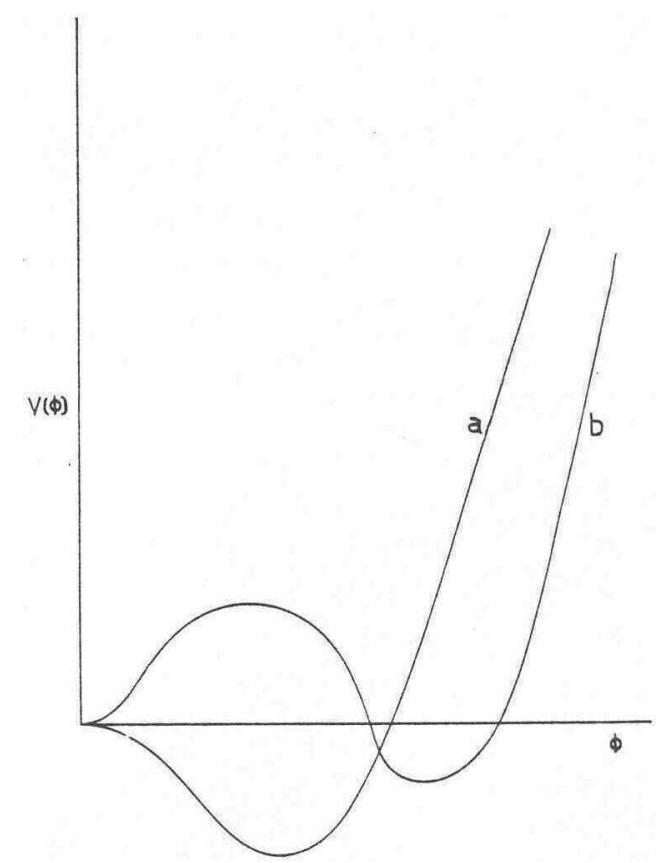

Fig. 1. Curve (a) conventional situation; curve (b) model of this paper 
Typically, the difference in the behaviour of the potential as a function of the field strength is sketched in fig. 1. Actually, the value of the potential $b$ at the minimum does not need to be negative, but can be adjusted, and in fact can be made zero. At the origin the curvature is opposite to that of the conventional case; in formula this means that the masses of the Higgs particles are real (i.e. masses squared positive) also before spontaneous symmetry breaking. The fact that with a suitable choice of constants the potential is zero at the minimum means that the cosmological constant is zero also after symmetry breakdown.

Consider now the Lagrangian (6), and make the replacements

$$
\phi_{i} \rightarrow \phi_{i}+F \delta_{i 1}, \quad \chi_{i j} \rightarrow \chi_{i j}+H \delta_{i 1} \delta_{j 1} .
$$

$F$ and $H$ are the vacuum expectation values of $\phi_{1}$ and $\chi_{11}$ respectively. Note that due to the U(1) symmetry we may assume $F$ to be real. There are three types of terms that must be considered:

(i) The cosmological term, that is the term of zeroth order in the fields $\phi, \chi$,

(ii) The tadpole terms in the tree diagram approximation, i.e. the terms linear in the fields. At some extremum of the potential these terms must be zero.

(iii) The mass terms. These are the terms quadratic in the fields. After diagonalization the occurring coefficients are directly related to the masses of the Higgs particles, and in a true minimum of the potential, all these masses must be real.

The tadpole terms are zero in the tree diagram approximation provided the following equations are satisfied:

$$
\begin{aligned}
& -\mu-\lambda F^{2}+\frac{1}{2}\left(a+a^{\prime}\right) H^{2}+b H=0, \\
& -\mu^{\prime}-\lambda^{\prime} H^{2}+\frac{1}{2}\left(a+a^{\prime}\right) F^{2}+\frac{1}{2} b F^{2} / H=0 .
\end{aligned}
$$

We will assume these equations to hold. The cosmological term is found to be:

$$
\frac{1}{2} \lambda F^{4}+\frac{1}{2} \lambda^{\prime} H^{4}-\frac{1}{2}\left(a+a^{\prime}\right) H^{2} F^{2}-\frac{1}{2} b F^{2} H .
$$

The mass terms are:

$$
\begin{aligned}
& \text { Mass terms }=M_{\mathrm{I}}+M_{\mathrm{II}}, \\
& \begin{array}{l}
M_{\mathrm{I}}=-\frac{1}{2} \lambda F^{2}\left(\phi_{1}+\phi^{1}\right)^{2}-\frac{1}{2} \lambda^{\prime} H^{2}\left(\chi_{11}+\chi^{11}\right)^{2} \\
\quad+\frac{1}{2}\left(a+a^{\prime}\right) F H\left(\phi_{1}+\phi^{1}\right)\left(\chi_{11}+\chi^{11}\right) \\
\quad+b F\left(\phi_{1} \chi^{11}+\phi^{1} \chi_{11}\right)+\frac{1}{2} b H\left(\phi_{1}-\phi^{1}\right)^{2}-\frac{1}{2} b\left(F^{2} / H\right) \chi^{11} \chi_{11}, \\
M_{\mathrm{II}}=+A\left(\phi_{2} \chi^{21}+\phi^{2} \chi_{21}\right)-A(H / F) \phi^{2} \phi_{2}-A(F / H) \chi^{12} \chi_{12} \\
\quad-\frac{1}{2} F^{2}\left(a^{\prime}+(b / H)\right) \chi^{22} \chi_{22},
\end{array}
\end{aligned}
$$


with

$$
A=\frac{1}{2} a^{\prime} F H+b F .
$$

It is seen that the real parts of $\phi_{1}$ and $\chi_{11}$ mix and so do the imaginary parts. Writing

$$
\phi_{1}=\sqrt{\frac{1}{2}}(C+i D), \quad \chi_{11}=\sqrt{\frac{1}{2}}(X+i Y),
$$

one rewrites for $M_{\mathrm{I}}$ :

$$
\begin{aligned}
& M_{\mathrm{I}}=-\lambda F^{2} C^{2}+\left\{\left(a+a^{\prime}\right) F H+b F\right\} C X-\left(\frac{1}{4} b F^{2} / H+\lambda^{\prime} H^{2}\right) X^{2} \\
& \quad-(b / 4 H)\left(F^{2}+4 H^{2}\right)\{Y \cos \alpha+D \sin \alpha\}^{2}, \\
& \cos \alpha=\frac{F}{\sqrt{F^{2}+4 H^{2}}} .
\end{aligned}
$$

This shows that the combination $-Y \sin \alpha+D \cos \alpha$ has zero mass, and in fact this is the unphysical Goldstone ghost. The other field combination corresponds to a particle with mass

$$
m_{01}^{2}=+(b / 2 H)\left(F^{2}+4 H^{2}\right) .
$$

This shows that $b / H$ must be taken positive. Diagonalization with respect to $C$ and $X$ is somewhat more complicated, and the resulting expressions for the masses are not particularly transparent. The condition that both masses are real is found to be:

$$
4 \lambda \lambda^{\prime} H^{2}>\left\{\left(a+a^{\prime}\right) H+b\right\}^{2}-\lambda b F^{2} / H,
$$

where $\lambda, \lambda^{\prime}$, and $b$ are taken to be positive. Remember that we must already have $4 \lambda \lambda^{\prime}>\left(a+a^{\prime}\right)^{2}$, in order that the potential is positive for large values of the fields. The masses found are

$$
\begin{aligned}
& m_{02}^{2}, m_{03}^{2}=\frac{1}{2}\left\{\lambda F^{2}+\lambda^{\prime} H^{2}+\frac{1}{4} b F^{2} / H\right. \\
& \left.\quad \pm \sqrt{\left(\lambda F^{2}-\lambda^{\prime} H^{2}-\frac{1}{4} b F^{2} / H\right)^{2}+\left(\left(a+a^{\prime}\right) F H+b F\right)^{2}}\right\} .
\end{aligned}
$$

Consider now $M_{\mathrm{II}}$. We see that also $\phi_{2}$ and $\chi_{21}$ mix. $M_{\mathrm{II}}$ may be rewritten:

$$
M_{\mathrm{II}}=-A\left(B \phi^{2}-(1 / B) \chi^{12}\right)\left(B \phi_{2}-(1 / B) \chi_{12}\right)-\frac{1}{2}\left(a^{\prime} F^{2}+b F^{2} / H\right) \chi^{22} \chi_{22} \text {, }
$$

where $A$ has been defined before, and

$$
B=\sqrt{H / F} .
$$

This implies zero mass for one combination of the $\phi_{2}$ and $\chi_{12}$ fields, and for the combination shown the mass is:

$$
m_{-}^{2}=A\left(B^{2}+1 / B^{2}\right)=\left(\frac{1}{2} a^{\prime}+b / H\right)\left(H^{2}+F^{2}\right) .
$$

The zero-mass object is again the unphysical Goldstone. The mass of the $\chi_{22}$ field is: 


$$
m_{--}^{2}=\frac{1}{2}\left(a^{\prime}+b / H\right) F^{2} .
$$

Provided $b+a^{\prime} H>0, b>0$, and condition (23) is satisfied all the masses are real.

Let us now consider the question of the cosmological constant. If we choose our parameters such that (19) is zero then at least one of the two constants $\mu, \mu^{\prime}$ must be positive. Indeed, summing the eqs. (18) multiplied with $F^{2}$ and $H^{2}$ respectively, and using that (19) is zero we find

$$
\mu F^{2}+\mu^{\prime} H^{2}=\frac{1}{2} b H F^{2}>0 .
$$

We are now interested in the positivity properties of the potential. Using eqs. (18), (19) we can eliminate three parameters $\left(\mu, \mu^{\prime}\right.$ and $\left.b\right)$ from the Higgs potential which can now be written

$$
\begin{aligned}
V & =\frac{1}{2} \lambda\left(\left(\phi^{i} \phi_{i}\right)^{2}+\left(F^{4} / H^{2}\right) \chi_{i j} \chi^{i j}-\left(F^{2} / H\right) \chi^{i j} \phi_{i} \phi_{j}-\left(F^{2} / H\right) \chi_{i j} \phi^{i} \phi^{j}\right) \\
& +\frac{1}{2} \lambda^{\prime}\left(\left(\chi^{i j} \chi_{i j}\right)^{2}-H^{2} \chi^{i j} \chi_{i j}+2\left(H^{4} / F^{2}\right) \phi^{i} \phi_{i}-\left(H^{3} / F^{2}\right) \chi^{i j} \phi_{i} \phi_{j}\right. \\
& \left.-\left(H^{3} / F^{3}\right) \chi_{i j} \phi^{i} \phi^{j}\right)-\frac{1}{2} a\left(\phi^{k} \phi_{k} \chi^{i j} \chi_{i j}+H^{2} \phi_{i} \phi^{i}-H \chi^{i j} \phi_{i} \phi_{j}-H \chi_{i j} \phi^{i} \phi^{j}\right) \\
& -\frac{1}{2} a^{\prime}\left(\phi^{i} \phi_{j} \chi^{j k} \chi_{i k}+H^{2} \phi_{i} \phi^{i}-H \chi^{i j} \phi_{i} \phi_{j}-H \chi_{i j} \phi^{i} \phi^{j}\right) .
\end{aligned}
$$

Without loss of generality we may assume $\phi_{2}=0$, and $\phi_{1} \neq 0$ and real. As can be easily seen in the Lagrangian (6) the potential'is minimal with constant magnitude $|\chi|^{2}$ and $|\phi|^{2}$ if we take real $\chi_{11} \neq 0$, all other $x$ zero. Let these non-zero components be denoted by $\phi$ and $\mathrm{x}$ respectively. We find:

$$
\begin{aligned}
V & =\frac{1}{2} \lambda\left(\phi^{4}+\left(F^{4} / H^{2}\right) \chi^{2}-\left(2 F^{2} / H\right) \chi \phi^{2}\right) \\
& +\frac{1}{2} \lambda^{\prime}\left(\chi^{4}-H^{2} \chi^{2}+\left(2 H^{4} / F^{2}\right) \phi^{2}-\left(2 H^{3} / F^{2}\right) \chi \phi^{2}\right) \\
& -\frac{1}{2}\left(a+a^{\prime}\right)\left(\phi^{2} \chi^{2}+H^{2} \phi^{2}-2 H \chi \phi^{2}\right) .
\end{aligned}
$$

It can be seen by inspection that this potential has a turning point at $\chi=\phi=0$, and that at that point $V=0$. Let us assume that this turning point is a minimum. This means that the coefficients of $\chi^{2}$ and $\phi^{2}$ must be positive (this is equivalent to assuming that $\mu$ and $\mu^{\prime}$ in the Lagrangian (6) are both positive). This implies the following inequalities

$$
\lambda>\lambda^{\prime} H^{4} / F^{4}, \quad \lambda^{\prime}>\frac{1}{2}\left(a+a^{\prime}\right) F^{2} / H^{2} .
$$

From this we immediately see that

$$
V \geqslant \frac{1}{2} \lambda^{\prime}\left(\left(H^{2} / F^{2}\right) \phi^{2}-\chi^{2}\right)^{2}, \quad \text { equality if } \phi^{2}=\left(F^{2} / H\right) \chi \text { and } \chi=H \text {, or } 0 .
$$

Thus $V$ can only be zero at the origin and at the required vacuum expectation value $\phi=F, \chi=H$. This point is therefore an absolute minimum so that all the masses must be real. 
Condition (27) tells us that $\mu$ or $\mu^{\prime}$ (or both) must be positive, if the cosmological term is to vanish If only one is positive then we have a saddle point rather than a minimum at the origin and the potential drops below zero in some directions away from the origin In such a case one of the other turning points of the potential (29) will be the absolute minimum, and that turning point will define the vacuum We therefore conclude that for a symmetry breaking vacuum with zero cosmological terms there must be also a degenerate symmetry-conserving vacuum However for the symmetry-conserving vacuum we will obtain zero mass for the intermediate vector bosons with all the associated infrared troubles of the unbroken Yang-Mills theory These infrared divergences may be sufficient to alter the stability of this state If we believe that our present scheme somehow derives from a theory without Higgs particles then this may after all not be so unnatural

\section{Vector boson masses in the general case}

It is very easy to extend the treatment of sect 2 to Higgs particles of arbitrary $I$ spin A field of $I$-spin $\frac{1}{2} n$ is represented by a tensor with $n$ indices There are $\frac{1}{2} n$ or $\frac{1}{2}(n-1)$ possibilities with respect to the properties under $\mathrm{U}(1)$ transformations, which may be conveniently expressed by the use of tensors with upper and lower indices, symmetric in upper and lower and tracelesswith respect to any contraction of upper and lower indices For such a tensor we have

$$
\begin{aligned}
& \left(D_{\mu} \chi\right)_{i j \ldots}^{k l \ldots}=\left[\delta_{k^{\prime}}^{k} \delta l_{l^{\prime}}^{l} \delta_{i}^{i^{i^{\prime}}} \delta_{j}^{j^{\prime}} \ldots \partial_{\mu}\right. \\
& \quad+g W_{\mu}^{a} \delta_{k^{\prime}}^{k} \delta_{l^{\prime}}^{l}\left\{\left(-\frac{1}{2} i \tau^{a}\right)_{i}^{i^{\prime}} \delta_{j}^{j^{\prime}} \ldots+\delta_{i}^{i^{\prime}}\left(-\frac{1}{2} i \tau^{a}\right)_{j}^{j^{\prime}} \ldots+\ldots\right\} \\
& \left.\quad+g W_{\mu}^{a} \delta_{i}^{i^{\prime}} \delta_{j}^{j^{\prime}}\left\{\left(\frac{1}{2} i \tau^{a}\right)_{k^{\prime}}^{k} \delta_{l^{\prime}}^{l} \ldots+\delta_{k^{\prime}}^{k}\left(\frac{1}{2} i \tau^{a}\right)_{l^{\prime}}^{l} \ldots+\ldots\right\}\right] \chi_{i^{\prime} j^{\prime}}^{k^{\prime} l^{\prime}} \ldots .
\end{aligned}
$$

In here $a$ runs from 0 to 3 , and $W_{\mu}^{0} \equiv B_{\mu} g^{\prime} / g$. The components decoupling trom the e $\mathrm{m}$ field are those for which the inde $x$ value 2 occurs as many times up as down like for example

$$
\chi_{2111 \ldots 111}^{2111} \text {. }
$$

All these components are equal in magnitude We will not bother to work out the mass ratios for this general case but note only that the $\mathrm{Z}$ mass becomes relatively smaller as the number of upper and lower indices become more equal If there are as many upper as lower indices no contribution to the $\mathrm{Z}$ mass results This follows trivially from the tact that upper indices transform with $-w_{\mu}$, the matrix shown in eq (8)

The maximum contribution to the $\mathrm{Z}$ mass relative to the charged mass obtains when a tensor with only lower indices is employed The component decoupling from the e $\mathrm{m}$ field is the one where all the lower indices have the value 1 Suppose this component has the vacuum expectation value $H$ Taking for $\chi_{i i \ldots}$ the vacuum 
expectation value $H \delta_{i 1} \delta_{j 1} \ldots$ we have:

$$
\begin{aligned}
& \left(D_{\mu} \chi\right)_{111 \ldots}=-n(i / 2 c) g H Z_{\mu}, \\
& \left(D_{\mu} \chi\right)_{2111 \ldots}=\left(D_{\mu} \chi\right)_{121 \ldots}=\ldots=-\frac{1}{2} i g H\left(W_{1}+i W_{2}\right)_{\mu} .
\end{aligned}
$$

All other components of $D_{\mu} \chi$ are zero. It follows that

$$
\left|D_{\mu} \chi\right|^{2}=H^{2}\left(\frac{1}{4}\left(n^{2} g^{2} / c^{2}\right) Z^{2}+\frac{1}{4} n g^{2} W_{1}^{2}+\frac{1}{4} n g^{2} W_{2}^{2}\right) .
$$

The relation between $\mathrm{Z}$ mass and charged $\mathrm{W}$ mass is

$$
M_{0}^{2}=\left(n / c^{2}\right) M_{ \pm}^{2}=\left(2 I / c^{2}\right) M_{ \pm}^{2},
$$

where $I$ is the $I$-spin of the Higgs particle. Thus, by using a sufficiently high representation the $\mathrm{Z}$ mass may be made arbitrarily large.

\section{Experimental consequences}

For experimental purposes we will assume here that the physical Higgs particles are too heavy to be of interest with present day energies. Then the upshot of the preceeding discussion is the introduction of a new parameter $\beta$,defined by:

$$
\beta=M_{ \pm}^{2} / e^{2} M_{0}^{2} .
$$

Apart from a factor $\beta^{2}$ the cross section for muon type neutrino scattering on electrons is as in the Weinberg model [7]:

$$
\begin{aligned}
& \sigma=0.425 \beta^{2} E \cdot 10^{-44} A(s) \mathrm{cm}^{2}, \\
& \begin{aligned}
A(s) & =1-4 s^{2}+\frac{16}{3} s^{4} \quad \text { for } \nu_{\mu} \mathrm{e}, \\
& =1-\frac{4}{3} s^{2}+\frac{16}{3} s^{4} \quad \text { for } \bar{\nu}_{\mu} \mathrm{e},
\end{aligned} \\
& s=\sin \theta_{\mathrm{W}}, \quad E \text { is neutrino energy } \mathrm{m} \mathrm{MeV} .
\end{aligned}
$$

In the case of no higher $I$-spin than the value $I$ we have, from eq. (35):

$$
\beta \geqslant 1 / 2 I \text {. }
$$

The Wemberg model corresponds to $\beta=1$. Relative to the predictions of the Wemberg model all neutral current cross sections are now multiplied with a factor $\beta^{2}$. If the Wemberg angle is determined from the ratio of veto $\bar{\nu}$ scattering (see fig. 2) then $\beta^{2}$ equals the ratio of the observed cross section to the predictions of the Weinberg model for that specific value of the Weinberg angle. Other predictions, such as the relative amounts of vector and axial vector contributions remain as in the Weinberg model.

As to the Higgs particles, we observe that they are weakly coupled to the vector 


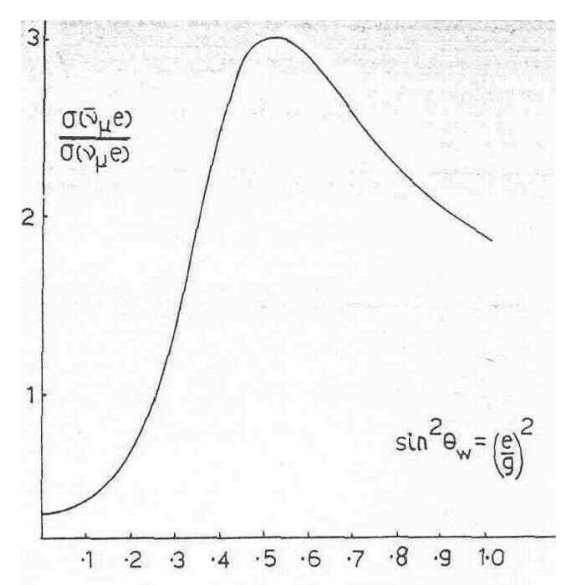

Fig. 2.

bosons, and therefore would not be easily detectable. To give the electron and muon a non-zero mass we need some $I=\frac{1}{2}$ Higgs scalars, but the resulting Yukawa couplings to electrons and muons are very small. An exception arises if the vacuum expectation value of the $I=\frac{1}{2}$ Higgs scalar is very small with respect to, say $I=1$ scalar. Then the Yukawa coupling may become rather strong, while also some of the physical Higgs masses become small (see eqs. (24), (26) for $F \sim 0$ ).

The presence of a doubly charged Higgs scalar has interesting consequences. It has no Yukawa coupling to fermions and so its decay is superweak, proceeding via two virtual $\mathrm{W}$ bosons. As such it could presumably have a lifetime long enough to produce a track in the appropriate detection instrument. However, its production cross section would be very small. It is amusing to note that if it were produced in a neutrino hadron scattering event it would convert the $\mathrm{W}^{+}$into a $\mathrm{W}^{-}$which could then produce a strange particle in apparent violation of the $\Delta S=\Delta Q$ rule (see fig. 3). We will not pursue this any further here.

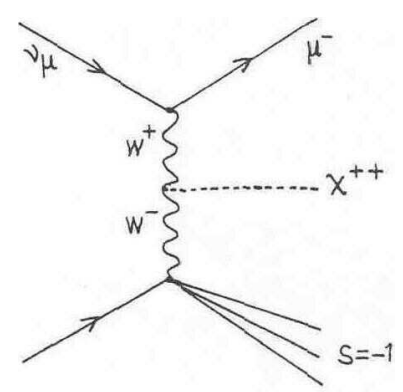

Fig. 3. 
The authors are indebted to Drs. G. 't Hooft and M. Lemoine for helpful discussions. This work is part of the research program of the "Stichting voor Fundamenteel Onderzoek der Materie" (F.O.M.), which is financially supported by the "Nederlandse Organisatie voor Zuiver Wetenschappelijk Onderzoek" (Z.W.O.).

\section{References}

[1] S.L. Glashow, Nucl. Phys. 22 (1961) 579.

[2] S. Weinberg, Phys. Rev. Letters 19 (1967) 1264.

[3] F. Englert and R. Brout, Phys. Rev. Letters 13 (1964) 321,

P.W Higgs, Phys. Letters 12 (1964) 132.

[4] A.D. Linde, ZhETF Pisma 19 (1974) 320; JETP Letters 19 (1974) 183;

M Veltman, to be published; Phys. Rev. Letters 34 (1975) 777;

J. Dreitlein, Phys. Rev Letters 133 (1974) 1243,

B. Zumino, CERN preprint 1974.

[5] T.D. Lee, Phys. Rev. D8 (1973) 1226.

[6] S. Wemberg, Phys. Rev. D7 (1973) 2887.

[7] G. 't Hooft, Phys. Letters 37B (1971) 195. 